\title{
Roughness Measurements on Coupling Structures for Optical Interconnections Integrated on a Printed Circuit Board
}

\author{
Nina Hendrickx ${ }^{1}$, Jürgen Van Erps ${ }^{2}$, Himanshu Suyal ${ }^{3}$, \\ Mohammed Taghizadeh ${ }^{3}$, Hugo Thienpont ${ }^{2}$, Peter Van Daele ${ }^{1}$ \\ ${ }^{1}$ Ghent University (UGent), TFCG Microsystems, Dept. of Information Technology (INTEC), \\ Ghent, Belgium \\ ${ }^{2}$ Vrije Universiteit Brussel (VUB), Dept. of Applied Physics and Photonics (TONA), \\ Brussels, Belgium \\ ${ }^{3}$ Heriot-Watt University (HWU), School of Engineering and Physical Sciences, Physics, \\ Edinburgh, United Kingdom
}

\begin{abstract}
In this paper, laser ablation (UGent), deep proton writing (DPW) and laser direct writing (HWU) are presented as versatile technologies that can be used for the fabrication of coupling structures for optical interconnections integrated on a printed circuit board (PCB). The optical layer, a highly cross-linked acrylate based polymer, is applied on an FR4 substrate. Both laser ablation and laser direct writing are used for the definition of arrays of multimode optical waveguides, which guide the light in the plane of the optical layer. In order to couple light vertically in/out of the plane of the optical waveguides, coupling structures have to be integrated into the optical layer. Out-of-plane turning mirrors, that deflect the light beam over $90^{\circ}$, are used for this purpose. The surface roughness and angle of three mirror configurations are evaluated: a laser ablated one that is integrated into the optical waveguide, a laser direct written one that is also directly written onto the waveguide and a DPW insert that is plugged into a cavity into the waveguiding layer.
\end{abstract}

Keywords: coupling structures, deep proton writing, laser ablation, laser direct writing, optical interconnects, optical waveguides, surface roughness

\section{Introduction}

It is predicted that the increase in microprocessor clock rate will create bandwidth limitations for copper interconnects on printed circuit boards (PCBs) due to signal attenuation, EMI/EMC problems, and crosstalk. Optical interconnections may overcome some of the bandwidth limitation of electrical interconnects on conventional PCBs. The integration of optical interconnections on a board-level, covering distances from a few $\mathrm{cm}$ to a couple of $\mathrm{m}$, can be done in a variety of ways. One of the possible approaches is the integration of an optical layer on a PCB. The optical layer, that contains optical waveguides and possibly also other passive optical structures, may be an alternative for the transmission of high data-rate, high-density signals.

One of the most critical problems is the coupling of light in and out of the optical plane. A common approach is the use of $45^{\circ}$ micro-mirrors. We investigate the use of laser ablation, laser direct writing and deep proton writing (DPW) for the definition of these micro-mirrors. Laser ablation and direct laser writing allow for the direct integration of these micro-mirrors with the waveguides in the optical layer. An alternative approach makes use of a pluggable out-of-plane coupling component with an integrated micro-mirror that can be readily inserted in laser ablated cavities. This type of pluggable component can be fabricated using Deep Proton Writing.

Surface roughness measurements have been performed with a non-contact optical profiler (WYKO NT-2000, available at VUB) on multimode optical waveguides defined with laser ablation and laser direct writing and micro-mirrors

Micro-Optics, VCSELs, and Photonic Interconnects II: Fabrication, Packaging, and Integration,

edited by H. Thienpont, M. R. Taghizadeh, P. Van Daele, J. Mohr, Proc. of SPIE Vol. 6185

61851E, (2006) - 0277-786X/06/\$15 - doi: 10.1117/12.665387

Proc. of SPIE Vol. 6185 61851E-1 
defined with all three available technologies. The surface roughness is a critical optical property since it is responsible for scattering loss that increases the propagation loss of the waveguides and decreases the coupling efficiency of the coupling structures. The surface profile of the laser ablated and laser direct written micro-mirrors are measured with a stylus contact surface profiler (Dektak, available at VUB). A comparative study will be made of all fabricated components.

\section{Fabrication technologies}

\subsection{Laser ablation}

Laser ablation is a flexible technology that can be used for the structuring of a large variety of materials. The technique is compatible with standard PCB manufacturing, and is already used for the laser drilling of micro-vias in high density boards. It is a non-mask based technology that can be used for rapid prototyping, as opposed to mask-based approaches where a mask first has to be designed and produced before the optical layer can be patterned. The ablation set-up available at UGent contains three different laser sources: a KrF excimer $(248 \mathrm{~nm})$, a frequency tripled Nd-YAG $(355 \mathrm{~nm})$ and a $\mathrm{CO}_{2}(9.6 \mu \mathrm{m})$ laser. Because of the availability of the different laser sources, a large variety of materials can be structured such as polymers, ceramics and glass. The excimer laser beam can be tilted, which eases the definition of angled facets considerably. The sample is during the processing placed on a computer-controlled translation stage, which has an accuracy of $1 \mu \mathrm{m}$. The three laser sources are available on the same set-up; as a consequence, the sample can stay on the same stage even when different sources are used for the patterning of the material. This approach allows us to achieve high alignment accuracies.

The $\mathrm{KrF}$ excimer laser $(248 \mathrm{~nm})$ is used for the definition of the multimode waveguides and metallized $45^{\circ}$ micromirrors in the optical layer. The multimode waveguides are defined by structuring of the waveguide core features into the core layer. These arrays of waveguides typically have a cross-section of $50 \times 50 \mu \mathrm{m}^{2}$ on a pitch of either $250 \mu \mathrm{m}$ or $125 \mu \mathrm{m}$. The definition of the metallized $45^{\circ}$ facet requires three processing steps: ablation of the $45^{\circ}$ facet, metallization of the facet, and filling of the ablated area with cladding material.

\subsection{Deep Proton Writing}

Deep Proton Writing (DPW) is a unique technology for the rapid prototyping of micro-mechanical and micro-optical elements ${ }^{1}$. The DPW fabrication process consists of the following basic steps.

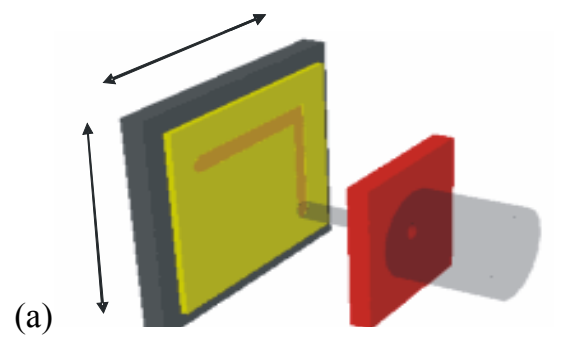

(b)

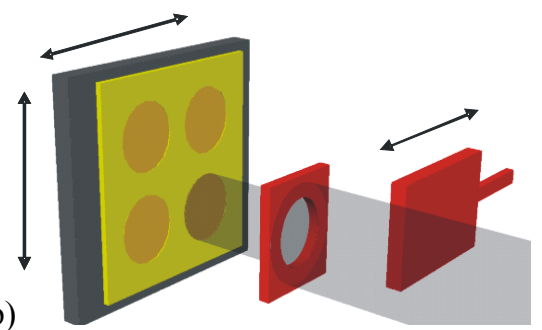

Figure 1: Irradiation step of DLP: Continuous (a) or point (b) irradiation of a PMMA sample

First a collimated $8.3 \mathrm{MeV}$ proton beam is used to irradiate an optical grade PMMA sample according to a predefined pattern by translating the PMMA sample, changing the physical and chemical properties of the material in the irradiated zones (Figure 1). As a next step, a selective etching solvent is applied for the development of the irradiated regions. This allows for the fabrication of ( $2 \mathrm{D}$ arrays of) micro-holes, optically flat micro-mirrors and micro-prisms, as well as alignment features and mechanical support structures. On the other hand, an organic monomer vapour can be used to expand the volume of the bombarded zones through an in-diffusion process. This enables the fabrication of spherical (or cylindrical) micro-lenses with well-defined heights. These processes are shown in Figure 2. If necessary, both processes can be applied to different regions of the same sample, yielding micro-optical structures combined with monolithically integrated micro-lenses. 


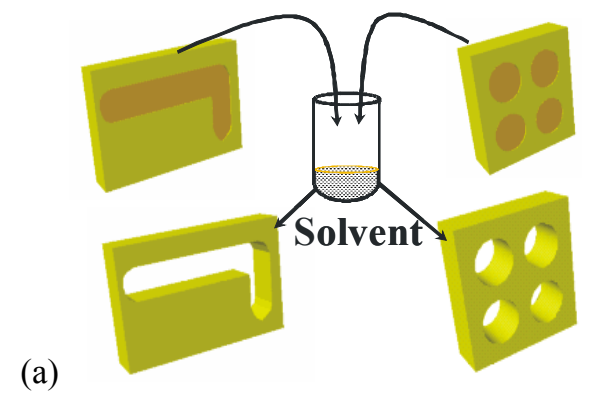

Figure 2: Chemical process steps of DLP: Selective etching process (a) and swelling process (b)

The coupling component is in this case a pluggable PMMA component with an integrated $45^{\circ}$ micro-mirror. The insert is placed in a cavity in the optical layer to couple light vertically in/out of a waveguide defined in the optical layer ${ }^{2}$. For the irradiation, we use high molecular weight PMMA with a thickness of $500 \mu \mathrm{m}$, which allows the $8.3 \mathrm{MeV}$ protons to completely traverse the sample. The PMMA sample is continuously translated perpendicularly to the beam in steps of $500 \mathrm{~nm}$ using Burleigh inchworms with an accuracy of 50nm. PMMA is a negative resist, which means that protons impinging on it will cause scissions in the long polymer chains and thus a material degradation in the irradiated zones, and that during the etching process the irradiated zones will be removed. This means that we have to irradiate the entire contour of the designed component. We have chosen to use a proton beam collimating aperture of $125 \mu \mathrm{m}$, which causes some rounding in the corners of the component, as shown in Figure 3, but this does not affect its optical functionality in any way.

(a)

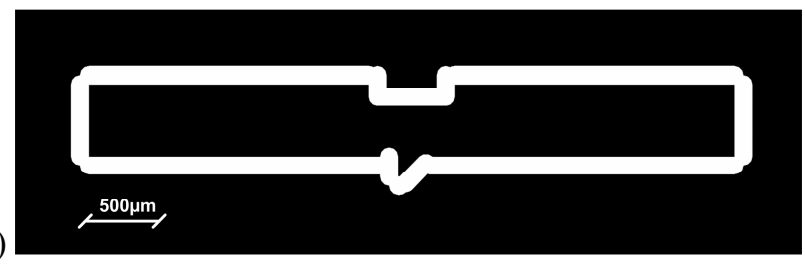

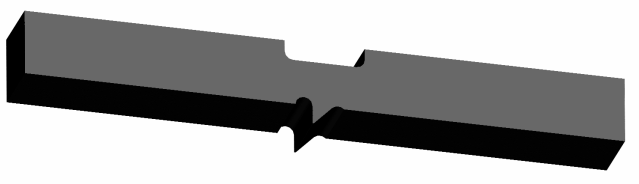

(b)

Figure 3: (a) Contour of the out-of-plane coupler as written by a $125 \mu \mathrm{m}$ proton beam and (b) the resulting component after etching

We have optimized the fabrication process as such that we achieve a minimal roughness of our fabricated optical surface. The best results are obtained when we irradiate the sample with a dose of $60 \mathrm{pC}$ per step at a beam current of $160 \mathrm{pA}$ and etch the irradiated sample during 1 hour in so-called GG developer at $38^{\circ} \mathrm{C}$, while using an ultrasonic stirrer. We fabricated two components, with exactly the same design.

\subsection{Laser direct writing}

To quote Laser Focus World, laser direct-write is a general term that encompasses modification, subtraction, and addition processes capable of creating patterns of materials directly on substrates without the need for lithography or masks. Laser direct-writing is a good alternative to photolithography for rapid prototyping as it is a maskless process. The laser direct writing system (HWU) is based on a He-Cd UV laser $(325 \mathrm{~nm})$ and a slotted base-plate which is used to align the polarizing, routing and focusing optics that guide and focus three beams (at normal and $\pm 45^{\circ}$ angles) onto a computer-controlled XY translation stages. Resolution of the XY translation stages is $1 \mu \mathrm{m}$. A flat-top intensity profile for the direct writing of the polymer is achieved by placing a $\sim 60 \mu \mathrm{m}$ square aperture on the axis of the $\sim 1.0 \mathrm{~mm}$ diameter $\mathrm{TEM}_{00}$ Gaussian beam and imaging it onto the polymer using two $80 \mathrm{~mm}$ focal length lenses. A substrate carrying a thin $(\sim 50 \mu \mathrm{m})$ layer of liquid photo-polymer solution is scanned beneath a focused spot of UV radiation ( $\sim 50 \mu \mathrm{m}$ across) to fabricate laser direct-written structures in polymer. The UV illuminated areas are polymerized, thus creating the required features and the rest of the uncured polymer is washed off during the development process.

The structures with vertical sidewalls (such as waveguide cores) can be fabricated using a beam at normal incidence and structures with angled sidewalls can be fabricated using non-normal beams. If a laser beam is incident on air/polymer interface it is impossible to get an angle of refraction $45^{\circ}$ in the polymer (refractive index of polymer $\sim 1.56$ ) due to the 
refraction at the interface. To overcome this limitation an oil-immersion technique is employed. A $90^{\circ} / 45^{\circ}$ prism is attached to the (fixed) optical assembly whilst the substrate with a thin layer of liquid polymer is placed in a shallow oilbath, mounted on the XY translation stage. In this way a $45^{\circ} \mathrm{UV}$ writing beam can be focused directly into the polymer layer with minimum deflection. The oil-immersion of the substrate during the writing provides an additional benefit of increased sensitivity of the polymer to the UV-beam by minimizing the inhibition effect which oxygen has on the polymerization process. Metallized $45^{\circ}$ facets are used for out-of-plane coupling. The substrate, coated with the liquid photo-polymer, is immersed in oil so that it is possible to get the laser beam at $45^{\circ}$ inside the polymer for the definition of $45^{\circ}$ angled features using the non-normal beams. The $45^{\circ}$ facets are then selectively metallized using evaporation through a shadow mask. The optical multimode connecting waveguides are laser direct written in a next step to complete the mirror fabrication.

\section{Roughness measurements}

\subsection{Optical multimode waveguides}

Surface roughness measurements have been performed on the top surface of an array of uncladded multimode waveguides defined with both laser ablation and laser direct writing. Measurements are performed on a sample that contains multiple arrays and the resulting RMS surface roughness is averaged.

\subsubsection{Laser ablation}

The results presented in Fig. 4 show that the top surface of the waveguide core has a RMS surface roughness of $\sim 35 \mathrm{~nm}$ on a scan area of $60 \mu \mathrm{m} \times 46 \mu \mathrm{m}$. An SEM image of a laser ablated waveguide core and a cross-section of an array of laser ablated multimode waveguides is also shown.

\subsubsection{Laser direct writing}

In Fig. 5, an SEM image is shown of an array of uncladded laser direct written waveguide cores and a cross-section of the end facet of a laser direct written waveguide. The results presented in Fig. 6 show that the top surface of the waveguide core has a RMS surface roughness of $\sim 227 \mathrm{~nm}$ on a scan area of $61 \mu \mathrm{m} \times 33 \mu \mathrm{m}$. Some lines are visible in the surface profile, originating from the laser direct writing process.

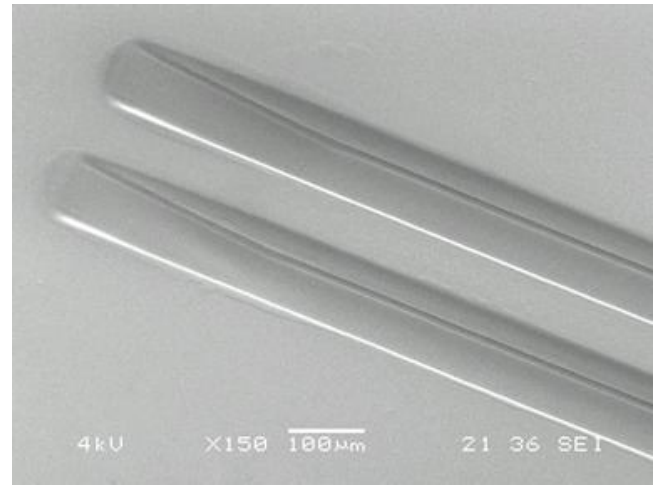

(a)

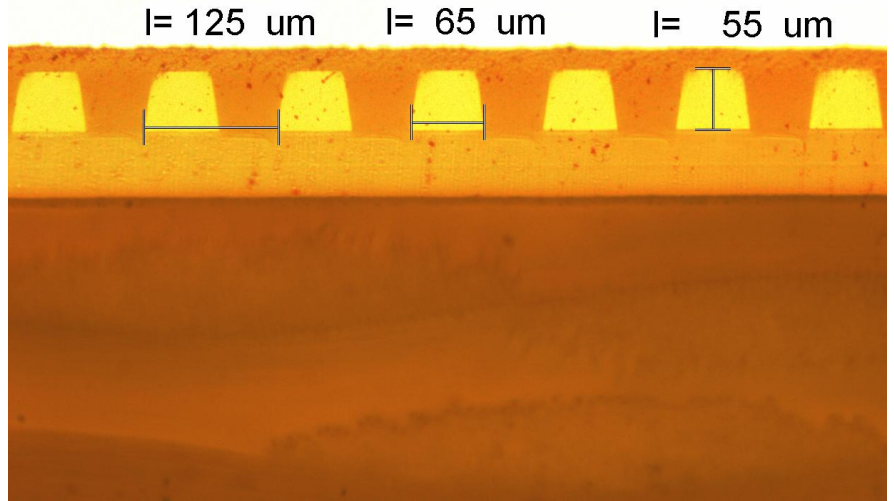

(b) 

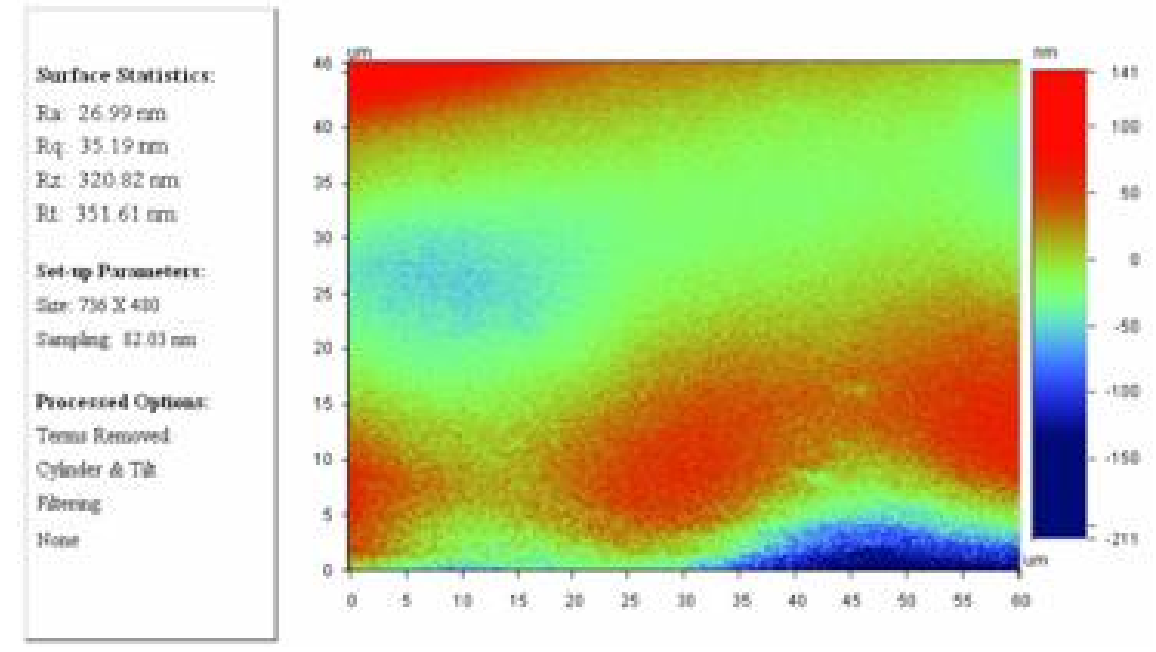

(c)

Figure 4: (a) SEM image of a laser ablated waveguide core; (b) cross-section of an array of laser ablated waveguides with a crosssection of $\sim 50 \times 50 \mu \mathrm{m}^{2}$ on a pitch of $125 \mu \mathrm{m}$; (c) results from surface roughness measurements performed on an uncladded waveguide core.
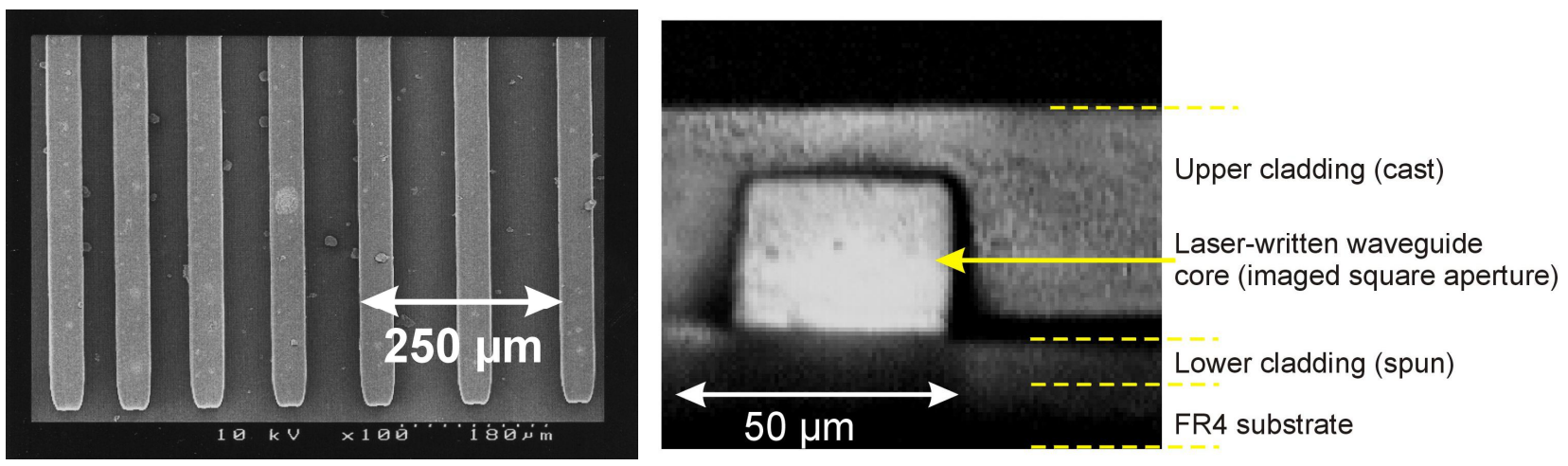

Figure 5: the left picture shows an SEM image of an array of laser direct written multimode waveguides on a pitch of $250 \mu \mathrm{m}$; the right picture shows the end facet of a laser direct written waveguide with a cross-section of $\sim 50 \times 50 \mu \mathrm{m}^{2}$.

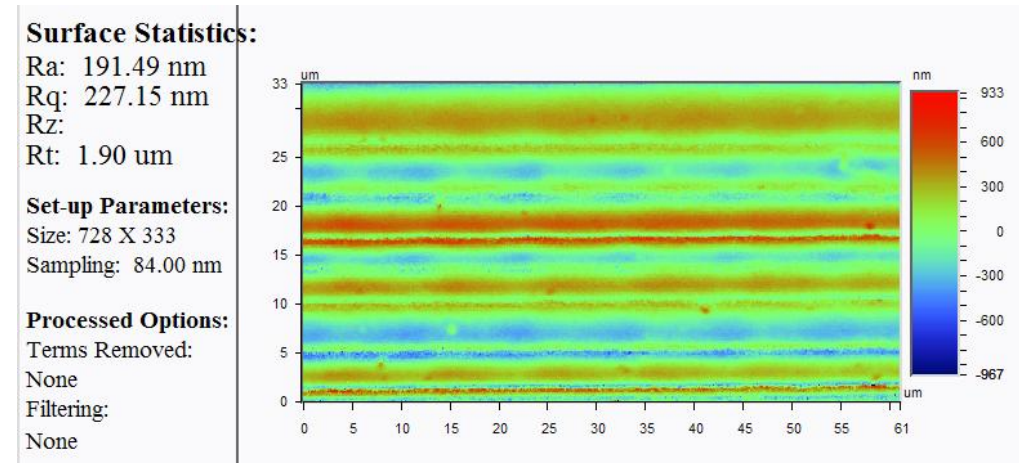

Figure 6: results from surface roughness measurements performed on the top surface of an uncladded laser direct written waveguide core, some lines that originate from the laser direct writing process can be observed in the surface plot. 


\subsection{Coupling structures}

\subsubsection{Laser ablation}

Both surface roughness measurements and profile measurements have been performed on the ablated $45^{\circ}$ facet. The results are presented in Fig. The $45^{\circ}$ angle of the micro-mirror has been confirmed by profile measurements, as shown in Fig. 7. The area of the micro-mirror that is used for out-of-plane coupling has an average RMS surface roughness of $53 \mathrm{~nm}$ on a scan area of $52 \times 174 \mu \mathrm{m}^{2}$.
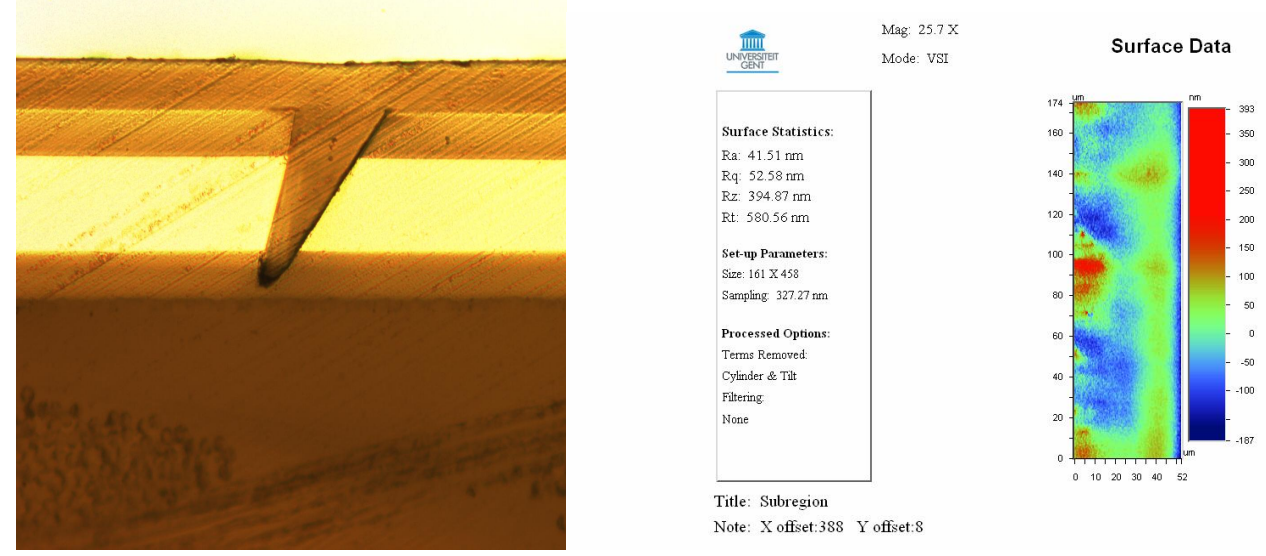

Figure 7: the left picture shows a cross-section of a metallized $45^{\circ}$ micro-mirror; the right picture shows the results from the surface roughness measurements performed on the ablated $45^{\circ}$ facet.

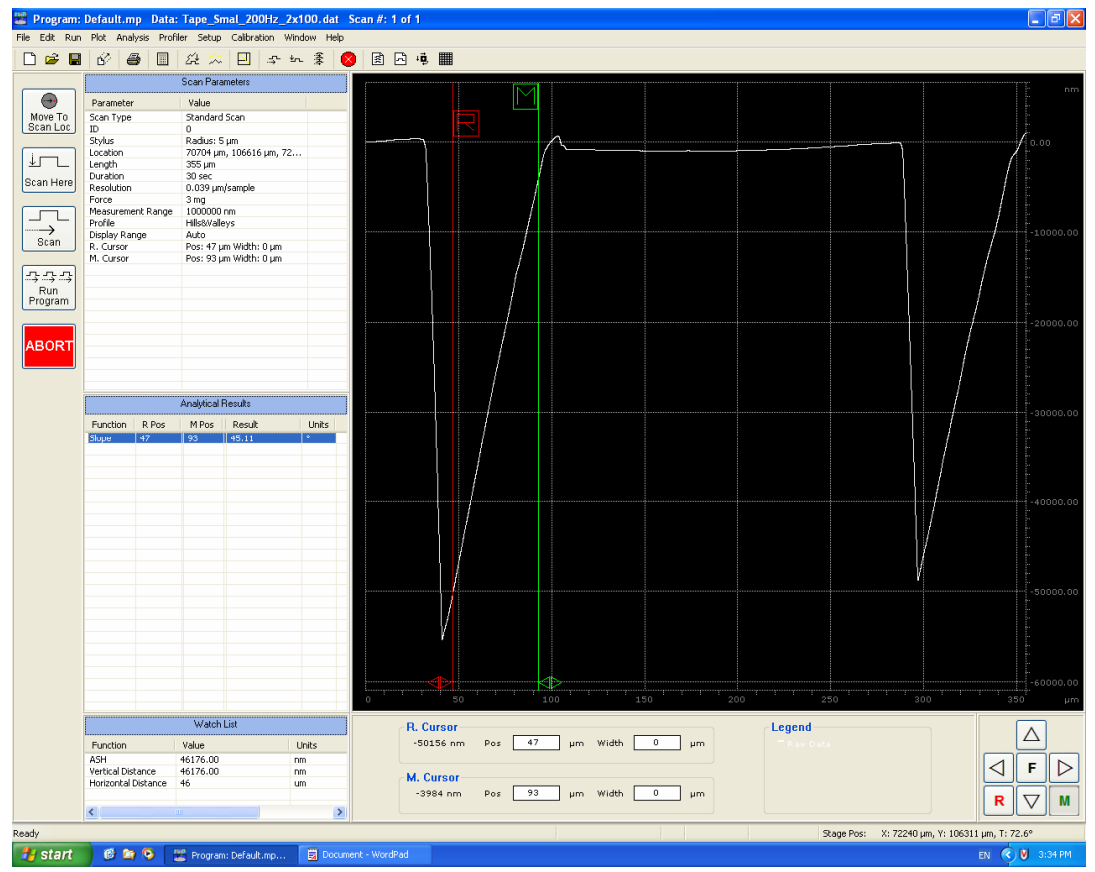

Figure 8: surface profile of a laser ablated $45^{\circ}$ facet, the measurement confirms the $45^{\circ}$ angle of the facet. 


\subsubsection{Deep Proton Writing}

Fig. 9 shows microscope images of the out-of-plane coupling components fabricated with DPW, and close-ups on the micro-mirror. For the characterization of the critical optical surfaces of the component, namely the flat top exit facet, the entrance facet and the $45^{\circ}$ mirror facet, we use a WYKO NT-2000 non-contact optical surface profiler. Since the entrance facet is not accessible with the microscope objective, this surface was not measured, but its surface roughness will be analogous to the two others. The surface analysis reveals that the flat top part has an average local RMS surface roughness $R_{q}$ of $34.19 \mathrm{~nm} \pm 4.90 \mathrm{~nm}$ (for component 1) and of $25.10 \mathrm{~nm} \pm 1.29 \mathrm{~nm}$ (for component 2), measured over an area of $60 \mu \mathrm{m}$ by $46 \mu \mathrm{m}$. We averaged 5 measurements of randomly chosen positions. Applying the same measurement method to the $45^{\circ}$ angled facet reveals an RMS roughness of $29.24 \mathrm{~nm} \pm 3.96 \mathrm{~nm}$ and of $29.21 \mathrm{~nm} \pm 3.96 \mathrm{~nm}$ for component 1 and 2, respectively. We can conclude that our developed surfaces have a very reproducible optical quality.

(a)
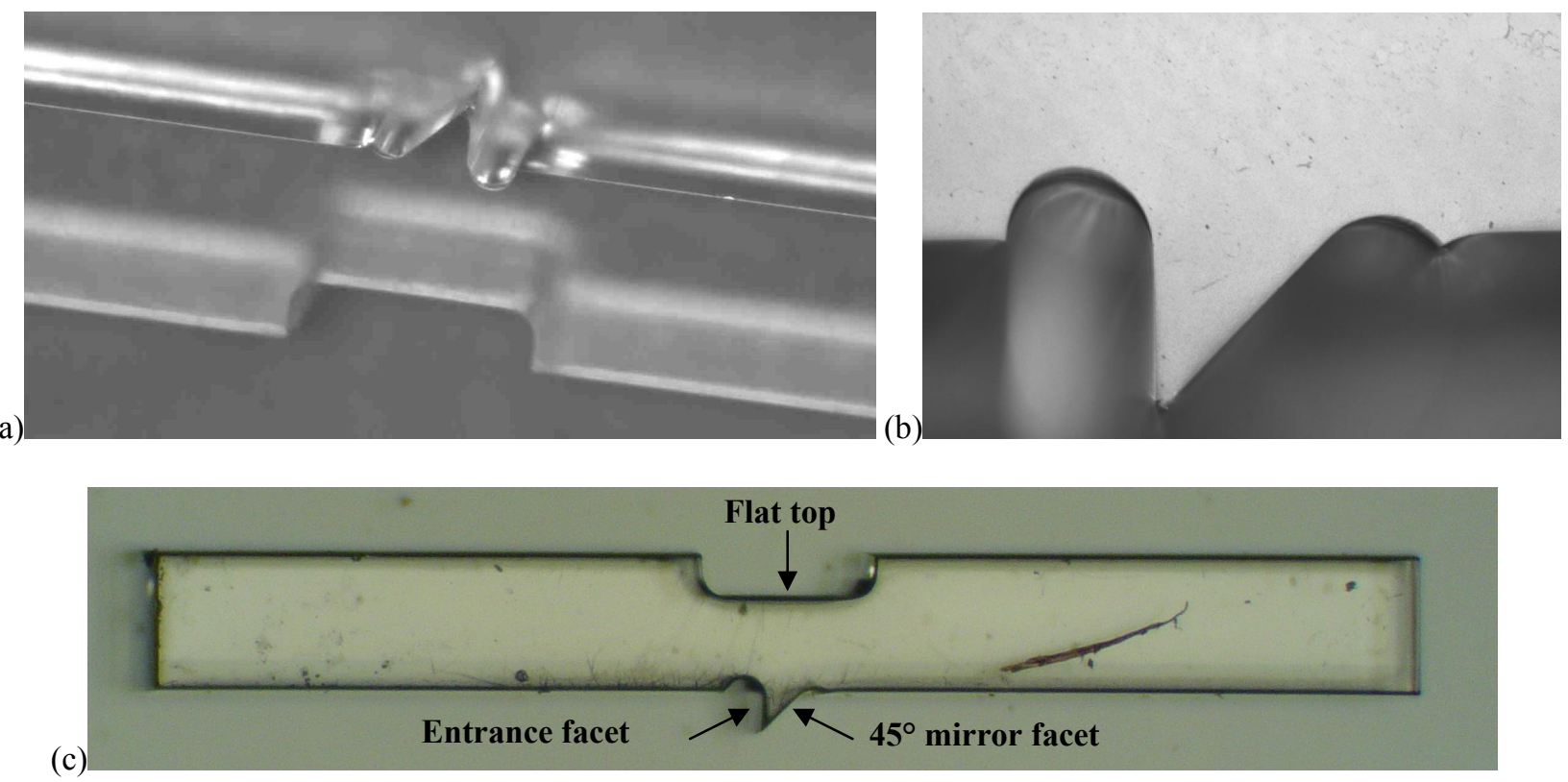

Figure 9: Stereo-microscope (a) and high-resolution microscope (b, c) images of the fabricated out-of-plane coupling components

The peak-to-valley flatness $R_{t}$ is $3 \mu \mathrm{m}$ over a length of $500 \mu \mathrm{m}$ in the direction of the proton path during the irradiation step. The latter is due to the scattering effect of the protons when interacting with the PMMA molecules. However, in the direction perpendicular to this path, the flatness $R_{t}$ is completely determined by the surface roughness, and thus in the nm range.

\subsubsection{Laser direct writing}

The profile of the angled structure has been measured with a stylus contact surface profiler (dektak). The results are shown in Fig. 10. The measured angle averaged $54^{\circ}$. Surface roughness measurements could not be performed on these mirrors using the non-contact surface profiler since the angle deviated too much from the desired $45^{\circ}$. 


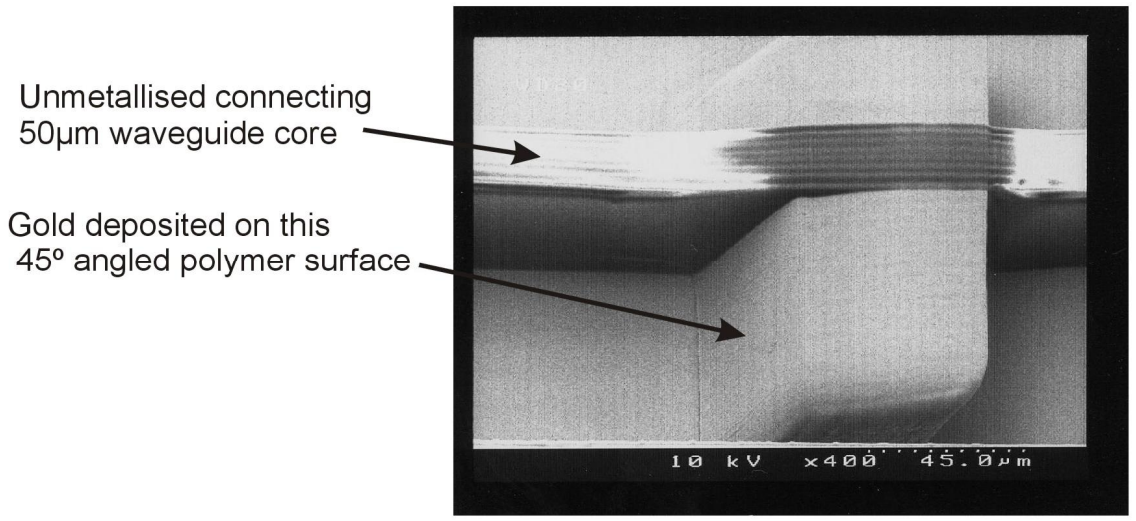

Figure 10: SEM image of an angled facet and a waveguide

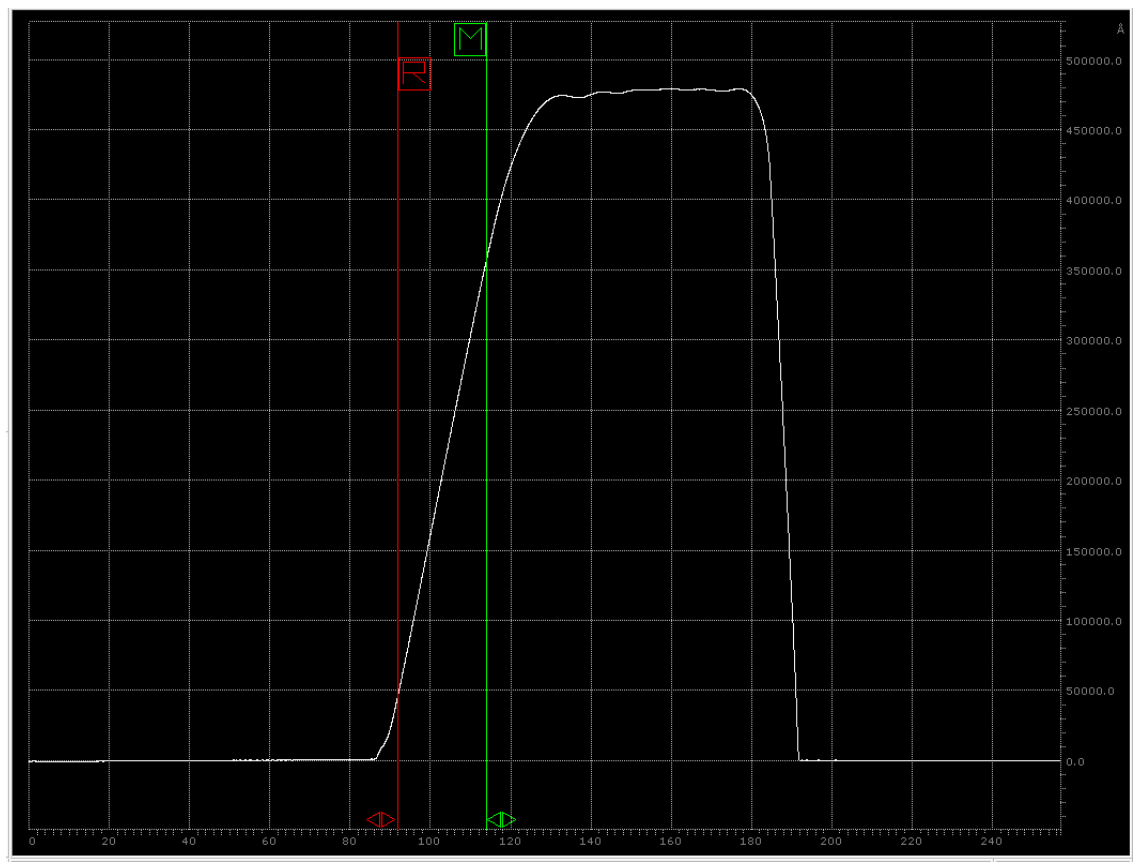

Figure 11: surface profile of an angled laser direct written facet, the measured angle is $54^{\circ}$

\subsection{Comparison}

In the following tables, the surface roughness measurements are summarized, for respectively waveguide sidewall surface roughness and coupling structure $\left(45^{\circ}\right.$ micro-mirror) roughness. Although no multimode waveguides have been fabricated with Deep Proton Writing, we have included the surface roughness measurements on the flat top part of the out-of-plane coupler in Table 1, since this can also be considered a sidewall roughness.

\begin{tabular}{|l|c|c|c|}
\hline WAVEGUIDES & Laser Ablation & Laser Direct Writing & Deep Proton Writing \\
\hline Surface roughness $\mathbf{R}_{\mathbf{q}} \mathbf{( n m )}$ & $35 \mathrm{~nm}$ & $227 \mathrm{~nm}$ & $29.65 \mathrm{~nm} \pm 4.90 \mathrm{~nm}$ \\
\hline Measured area & $60 \mu \mathrm{m} \times 46 \mu \mathrm{m}$ & $61 \mu \mathrm{m} \times 33 \mu \mathrm{m}$ & $60 \mu \mathrm{m} \times 46 \mu \mathrm{m}$ \\
\hline Terms removed & Tilt \& Cylinder & None & Tilt \\
\hline
\end{tabular}

Table 1: Surface roughness measurements on waveguide sidewall 


\begin{tabular}{|l|c|c|c|}
\hline COUPLING STRUCTURES & Laser Ablation & Laser Direct Writing & Deep Proton Writing \\
\hline Surface roughness $\mathbf{R}_{\mathbf{q}}(\mathbf{n m})$ & $52.58 \mathrm{~nm}$ & x & $29.2 \mathrm{~nm} \pm 3.96 \mathrm{~nm}$ \\
\hline Measured area & $174 \mu \mathrm{m}$ x $52 \mu \mathrm{m}$ & x & $60 \mu \mathrm{m}$ x $46 \mu \mathrm{m}$ \\
\hline Terms removed & Tilt \& Cylinder & x & Tilt \\
\hline
\end{tabular}

Table 2: Surface roughness measurements on coupling structures, i.e. $45^{\circ}$ micro-mirrors

A direct comparison of RMS roughness $\mathrm{R}_{\mathrm{q}}$ should be done with the necessary precaution. The area measured and the terms removed from the measurement influence the value of $\mathrm{R}_{\mathrm{q}}$ strongly and that is why they have been included in the summarizing tables. Keeping this in mind, it can be seen from Table 1 that laser ablation and deep proton writing offer a comparable sidewall roughness. Both technologies allow the fabrication of really optical surfaces with $\mathrm{R}_{\mathrm{q}}$ values of at least $\lambda / 25$ for the targeted datacom wavelength of $850 \mathrm{~nm}$. The $\mathrm{R}_{\mathrm{q}}$ for laser direct written structures is much higher, but it should be noticed that it is really the top wall of the waveguide core that has been measured, in which a line pattern is clearly visible. Removing tilt and cylinder does not improve the measured $\mathrm{R}_{\mathrm{q}}$. The 'real' sidewalls, however, might exhibit a lower roughness.

As far as the coupling structures are concerned, a comparison can only be made between laser ablation and deep proton writing (see Table 2), even though the measurements have been carried out over a different scan area. It should also be noted that the concept is completely different for both approaches, since in the case of laser ablation, the micro-mirror is written directly in the waveguide, whereas in the case of DPW, the micro-mirror is on a separate component, which can be readily plugged into cavities in a PCB-integrated waveguide layer.

\section{Conclusions}

In this paper, we have compared three technologies that enable the fabrication of multimode waveguides and/or coupling structures, more in particular out-of-plane turning $45^{\circ}$ micro-mirrors. Laser ablation, laser direct writing and deep proton writing all have specific advantages and disadvantages. With respect to resulting surface roughness of the fabricated components, we see that the result obtained with laser ablation and deep proton writing is comparable. A real comparison between the different components would however imply the measurement of a more integrated structure containing optical waveguides and the out-of-plane coupling component. In this way, the overall efficiency of the different systems can be compared. Measurements on this subject will be performed in the near future.

\section{ACKNOWLEDGEMENTS}

This work was carried out within the framework of the Network of Excellence in Micro-Optics (NEMO) supported by the European Commission through the SP6 program.

Nina Hendrickx would like to acknowledge the Institute for Promotion of Innovation by Science and Technology in Flanders (Flemish IWT) for financial support.

Jürgen Van Erps is indebted to the FWO (Fund for Scientific Research Flanders) for his research fellowship and financial support.
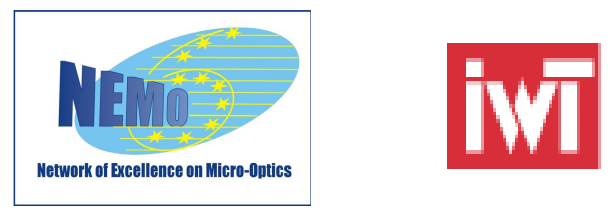

\section{REFERENCES}

1. B. Volckaerts et al., "Deep Lithography with Protons: a generic fabrication technology for refractive micro-optical components and modules", Asian J. of Physics, 10 (2), pp. 195-214, 2001. 
2. J. Van Erps, et al., "Deep lithography with protons to prototype pluggable micro-optical out-of-plane coupling structuresfor multimode waveguides", Proceedings of the SPIE, Internation Congress on Optics and Optoelectronics (ICOO '05), Vol 5956, pp. 52-63, October 2005

3. G. Van Steenberge, N. Hendrickx, E. Bosman, J. Van Erps, H. Thienpont, P. Van Daele, Laser Ablation of Parallel Optical Interconnect Waveguides, Photonics Technology Letters, accepted for publication 